Forum $2021 \cdot 36: 492-496$

https://doi.org/10.1007/s12312-021-00996-z

Online publiziert: 18 . Oktober 2021

๑) Springer Medizin Verlag GmbH, ein Teil von

Springer Nature 2021

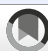

Check tor
updates

\section{Onkologische Versorgungsforschung in Deutschland}

\author{
Neue Perspektiven durch Vernetzung
}

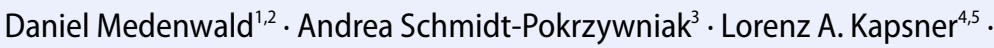
Nina Buttmann-Schweiger ${ }^{6}$. Sina Hopff $\cdot$ Heike Schmidt ${ }^{1,89} \cdot$. Dirk Vordermark ${ }^{1}$ 'Klinik und Poliklinik für Strahlentherapie, Universitätsklinikum Halle (Saale), Halle (Saale), Deutschland; ${ }^{2}$ AG Onkologische Versorgungsforschung, Klinik für Strahlentherapie, Martin-Luther-Universität HalleWittenberg, Halle (Saale), Deutschland; ${ }^{3}$ Klinisches Krebsregister Sachsen-Anhalt gGmbH, Magdeburg, Deutschland; ${ }^{4}$ Medizinisches Zentrum für Informations- und Kommunikationstechnik,

Universitätsklinikum Erlangen, Erlangen, Deutschland; ${ }^{5}$ Radiologisches Institut, Universitätsklinikum Erlangen, Friedrich-Alexander-Universität Erlangen-Nürnberg (FAU), Erlangen, Deutschland; ${ }^{6}$ Zentrum für Krebsregisterdaten, Robert Koch-Institut, Berlin, Deutschland; ${ }^{7}$ Medizinische Fakultät und Uniklinik Köln, Klinik I für Innere Medizin, Universität zu Köln, Köln, Deutschland; ${ }^{8}$ Institut für Gesundheits- und Pflegewissenschaft, Medizinische Fakultät, Martin-Luther-Universität Halle-Wittenberg, Halle (Saale), Deutschland; ${ }^{9}$ AG Lebensqualität, Klinik für Strahlentherapie, Martin-Luther-Universität HalleWittenberg, Halle (Saale), Deutschland
\end{abstract}

\section{Zusammenfassung}

\section{In diesem Beitrag}

- Forschungsfragen und methodische Ansätze

- Krebsregister

- Medizininformatik-Initiative

- Nationale Pandemie Kohorten Netz (NAPKON)

Neben der prospektiven Versorgungsforschung mit ihrem qualitativen und quantitativen Ansatz sind retrospektive Datenquellen essenziell zur Beurteilung von Prozessen im Gesundheitswesen. In Deutschland ermöglichen strukturelle Veränderungen in den Krebsregistern bzw. die Etablierung neuer Datenquellen interessante Möglichkeiten für die Versorgungsforschung. So wurde mit der Medizininformatik-Initiative eine Vernetzung klinischer Einrichtungen aufgebaut, womit sich schnelle, sektorübergreifende und lebensnahe Analysen realisieren lassen. Durch die Neustrukturierung der Krebsregister sollen bevölkerungsbasierte Erhebungen im onkologischen Bereich vereinfacht und die Detailtreue der Daten erhöht werden. Die Folgen der COVID-19-Pandemie (Coronavirus-Krankheit 2019) im onkologischen Bereich lassen sich dabei über das neu geschaffene Nationale Pandemie Kohorten Netz (NAPKON), ein Zusammenschluss universitärer und nichtuniversitärer Gesundheitsdienstleister, abbilden. Durch die Einbeziehung der Akteure im Gesundheitswesen und unter der Mitwirkung prospektiver Studien lässt sich damit ein umfassendes Bild des onkologischen Versorgungsgeschehens in Deutschland zeichnen.

\title{
Schlüsselwörter
}

Gesundheitswesen · Krebsregister · Medizininformatik-Initiative · Nationale Pandemie Kohorten Netz $\cdot$ COVID-19. Versorgungsforschung

Die Versorgungsforschung nimmt in den letzten Jahren eine zentrale Rolle in der praxisnahen Analyse onkologischer Erkrankungen ein. Die Heterogenität der Patient*innen hinsichtlich Bedarfen sowie eine zunehmende Bedeutung des ambulanten Sektors bei multimodalen Therapiekonzepten führt zu neuen Herausforderungen. Gleichzeitig wächst die
Zahl älterer Patient*innen [1-3]. Dies begründet die Notwendigkeit eines breiten Methodenspektrums in der Versorgungsforschung.

Die Versorgungsforschung versucht auf der Grundlage verschiedener Datenquellen die Qualität im Prozess der medizinischen Leistungserbringung zu beurteilen (Input-Throughput-Modell; [4]). 
Im Bereich der prospektiven Studien lässt sich ein qualitatives von einem quantitativen Vorgehen unterscheiden. Der qualitative Ansatz erlaubt dabei eine breite Untersuchung relevanter Fragestellungen.

Demgegenüber wurden in den letzten Jahren besonders für retrospektive Ansätze neue Datenquellen erschlossen. Durch die breite Zweckbindung und einen recht niedrigschwelligen Datenzugang zu standardisiert erhobenen Datensätzen bilden die Krebsregister traditionell den wichtigsten Ansatzpunkt zur Untersuchung von Krebserkrankungen auf einer bevölkerungsbasierten Ebene. Mit der Medizininformatik-Initiative (MII) und dem Nationalen Pandemie Kohorten Netz (NAPKON) zur sektorübergreifenden Untersuchung der COVID-19-Pandemie (CoronavirusKrankheit 2019) stehen aktuell neue sektorübergreifende Datengrundlagen zur Verfügung.

Die gesamtgesellschaftliche Bedeutung der Versorgungsforschung für die onkologische Therapielandschaft in Deutschland wurde in einem Memorandum des Deutschen Netzwerks Versorgungsforschung e. V. herausgestellt [5].

Ziel des Artikels ist es, die beiden methodischen Stränge der retrospektiven bzw. prospektiven Methoden der onkologischen Versorgungsforschung zu beleuchten und wichtige Datenquellen zur Versorgungsforschung in Deutschland exemplarisch zu benennen.

\section{Forschungsfragen und methodische Ansätze}

Im Folgenden sollen zunächst beispielhaft mögliche wissenschaftliche Fragestellungen sowie methodische Ansätze vorgestellt werden, um Defizite zu identifizieren und Konzepte zur Optimierung der Versorgung zu entwickeln.

Routinedaten können in Zusammenschau mit Selbstauskünften der Patient*innen („patient-reported outcomes“) herangezogen werden, um Langzeitoutcomes wie Lebensqualität und Funktionalität zu analysieren. Ergänzend können qualitative Daten Erkenntnisse liefern, wie vorhandene Strukturen genutzt werden und welche strukturellen und individuellen Faktoren die optimale Nutzung verfügbarer Ressourcen beeinflussen. Aus Sicht der Patient*innen können Erkenntnisse zu möglichen Gründen gewonnen werden, warum medizinische Leistungen (nicht) in Anspruch genommen bzw. Behandlungen abgebrochen werden [2]. Weitere Untersuchungsgegenstände könnten in dem Zusammenhang die Bedeutung zielgruppengerechter Aufklärung, partizipativer Entscheidungsfindung, multiprofessionelle Therapie, Einbeziehung von Angehörigen und Beratungsstellen sowie Selbsthilfegruppen in den Versorgungsprozess sein [3].

Ein Zusammenwirken quantitativer und qualitativer Methoden, im Rahmen von Mixed-methods-Studien, kann helfen, den Versorgungsprozess aus unterschiedlichen Perspektiven und in unterschiedlichen Settings zu verstehen und Optimierungsbedarfe zu identifizieren [6]. Nach Niederberger und Peter können folgende Studiendesigns zur Anwendung kommen [6]:

- Gleichzeitige Durchführung quantitativer und qualitativer Erhebungen, Ergebnisse werden in Zusammenhang gesetzt (Mixed-methods-Studien mit parallelem Triangulationsdesign)

- Abfolge von qualitativer Hypothesen-generierender Vorstudie und Hypothesen-prüfender quantitativer Folgestudie („exploratory sequential design")

- Durchführung einer quantitativen Studie gefolgt von einer qualitativen Studie zur Vertiefung unerwarteter Ergebnisse und Identifikation weiterer Fragestellungen („explanatory sequential design")

Folglich erlauben qualitative Methoden die Identifikation von möglichen Ursachen in quantitativen Vergleichen.

Geeignete Verfahren für die Erhebung qualitativer Daten sind z. B. offene oder teilstrukturierte Fokusgruppeninterviews, individuelle Interviews oder schriftliche Befragungen, deren Daten mittels inhaltsanalytischer Verfahren, z.B. nach Mayring, analysiert werden können [7]. Zur Einbeziehung von Betroffenen werden häufig Selbsthilfegruppen adressiert.

Weitere methodische Grundlagen liefert das Framework des Medical Research Council zur Entwicklung und Evaluation komplexer Interventionen [8,9]. Unter Einbeziehung aller Beteiligten sollte auf Basis der gewonnenen Erkenntnisse zunächst theorie- und evidenzgeleitet eine gezielte Auswahl der wichtigsten Strategien erfolgen. Vor einer Implementierung sollten die Prozesse modelliert und das neue Vorgehen pilotiert werden, um Machbarkeit, Akzeptanz und potenziellen Nutzen zu prüfen [9]. Ergänzend werden methodische Grundlagen für folgende Fragen der Implementierung in die reale Versorgungslandschaft im Consolidated Framework for Implementation Research umfänglich adressiert [10].

Damit ist in der Versorgungsforschung eine Methodenvielfalt von größter Bedeutung für die Identifikation von Optimierungsbedarfen, ein vertieftes Verständnis möglicher Problemfelder und Ursachen bis zur Entwicklung von Lösungsstrategien und der Implementierung neuer Versorgungskonzepte.

\section{Krebsregister}

Im Jahr 2013 wurde das Krebsfrüherkennungs- und -registergesetz (KFRG) verabschiedet, welches über eine Änderung des Fünften Buches Sozialgesetzbuch (SGBV) alle Bundesländer verpflichtete, flächendeckende klinische Krebsregister einzurichten. Das Hauptziel des KFRG ist es, Verbesserungspotenziale in der Krebsbehandlung und -versorgung aufzudecken und die Grundlagen für geeignete Verbesserungsmaßnahmen bereitzustellen. Durch eine weitere Änderung des SGBV vom August 2021 [11] sind nunmehr die Aufgaben der klinischen Krebsregister u.a. um eine Übermittlung von klinischen Daten an das Zentrum für Krebsregisterdaten (ZfKD) erweitert worden. Bereits seit vielen Jahren tragen die am ZfKD bundesweit zusammengeführten, bevölkerungsbezogenen Registerdaten dazu bei, den Forschungsbedarf aufzuzeigen, Maßnahmen der Krebsbekämpfung, der Primär-und Sekundärprävention auf Bevölkerungsebene zu priorisieren sowie den Erfolg dieser Maßnahmen zu objektivieren [12].

Mit dem Gesetz zur Zusammenführung der Krebsregisterdaten [11] wurde die Erweiterung der zentral vorgehaltenen Krebsregisterdaten um Therapieangaben und den Krankheitsverlauf beschlossen, womit künftig der Blick in das bundesweite Versorgungsgeschehen krebskranker 
Menschen in Deutschland eröffnet wird. In einem zentralen Datensatz werden ab 2023 die Daten von der Diagnose über einzelne Behandlungsschritte, Remissionen bis hin zu Rezidiven und Mortalität für wissenschaftliche Zwecke verfügbar gemacht. Diese erweiterte Datenbasis und der um ein Jahr verkürzte Verzug der Daten eines Diagnosejahres (auf zwei bis drei Jahre) stellt eine herausragende Stärkung der registerbasierten Forschung in Deutschland dar.

Darüber hinaus schafft der Gesetzentwurf die Grundlage für eine weitere Ausbaustufe: Bundesweit soll künftig anlassbezogen, registerübergreifend die Zusammenführung der Basisdatensätze der Länder für wissenschaftliche Analysen der Daten ermöglicht werden. Hiermit nimmt die Aktualität der Auswertungen weiter zu, und es wird eine deutlich umfangreichere Datenbasis zu den an Krebs erkrankten Personen sowie zu Angaben der Meldenden zugänglich gemacht. Das ZfKD entwickelt vor diesem Hintergrund eine zentrale Antrags- und Registerstelle für entsprechende Nutzungsanträge. In der zweiten Ausbaustufe soll auch die Verknüpfung von Krebsregisterdaten mit zusätzlichen, den Registern nicht vorliegenden Daten (beispielsweise aus Studien) möglich werden. Bis zum Ende des Jahres 2024 werden die Krebsregister, das ZfKD und klinisch-wissenschaftliche Akteure aus Versorgung und Forschung gemeinsam mit Vertreterinnen und Vertretern von Patient*innenorganisationen ein Konzept für eine solche Plattformlösung erarbeiten [13].

Zu den Aufgaben der klinischen Krebsregister (vormals auch Landeskrebsregister) gehören die Erfassung der personenbezogenen Daten aller Patient*innen über das Auftreten, die Behandlung und den Verlauf von bösartigen Neubildungen, die Auswertung der erfassten klinischen Daten und Rückmeldung der Auswertungsergebnisse an Leistungserbringer sowie der Datenaustausch mit anderen regionalen klinischen Krebsregistern. Ein weiterer Schwerpunkt ist die Bereitstellung notwendiger Daten zur Herstellung von Versorgungstransparenz und zu Zwecken der Forschung sowie die Erarbeitung von Konzepten zur Erfassung des Krankheitsverlaufes.
Um diese Aufgaben ausführen zu können, hat das Gesetz verschiedene Meldeanlässe vorgesehen. Meldeanlässe sind Sachverhalte, welche eine Meldeverpflichtung auslösen. Folgende Meldeanlässe sind im Gesetz festgeschrieben:

- Krebsdiagnosestellung nach hinreichender klinischer Sicherung, histologische, zytologische oder labortechnische Sicherung,

- Beginn und Abschluss einer therapeutischen Maßnahme,

- jede Änderung im Verlauf einer Tumorerkrankung (z. B. Rezidiv, Metastasen),

- Ergebnis der Nachsorgeuntersuchungen inkl. einer jährlichen Meldung bei Tumorfreiheit (10 Jahre nach Abschluss der Therapie),

- Tod des/der Patient*in.

Zur Meldung verpflichtet ist jeder Arzt, Zahnarzt und im Falle von Krankenhäusern der ärztliche Direktor. Auch die Meldeinhalte sind gesetzlich vorgeschrieben, sie entsprechen dem ADT-GEKID-Basisdatensatz und ihn ergänzende Module. Damit ist ein einheitlicher bundesweiter Standard geschaffen worden [14].

Laut Gesetz müssen Patient*innen darüber informiert werden, dass eine Meldung an das Klinischen Krebsregister (KKR) vorgenommen wird. Alle Patient*innen haben das Recht, der Speicherung seiner/ ihrer Daten im Landeskrebsregister zu widersprechen. Dieser Widerspruch erstreckt sich nicht auf die Daten, die für die epidemiologische Krebsregistrierung und die Abrechnung benötigt werden.

Die Finanzierung der KKR erfolgt durch die Krankenkassen (90\% der Betriebskosten). Seit 2014 zahlen die Krankenkassen für jede Krebsneuerkrankung (außer prognostisch günstiger nichtmelanotischer Hautkrebs) eine Registerpauschale, welche an Kriterien zur Qualitätsverbesserung geknüpft ist.

Die KKR werten Daten zur Meldequalität und klinischen Versorgung aus und spiegeln sie in Form von Rückmeldeberichten und im Rahmen von regionalen Qualitätskonferenzen an die meldenden Ärzt*innen zurück. Des Weiteren können die Meldenden Rückinformation über sämtliche im Krebsregister vorhandenen Daten zu den von ihnen behandelten Patient*innen erhalten.
Die Auf- und Umbauphase ist zum aktuellen Zeitpunkt in den meisten Registern weitestgehend abgeschlossen. Die daraus generierbaren Erkenntnisse können so zur Verbesserung der onkologischen Gesundheitsversorgung genutzt werden.

\section{Medizininformatik-Initiative}

Die vom Bundesministerium für Bildung und Forschung (BMBF) geförderte MII hat zum Ziel, durch die neuen Möglichkeiten, die sich im Rahmen der Digitalisierung im Gesundheitswesen bieten, neue Lösungsansätze zu erarbeiten. Diese sollten u.a. durch das standardisierte Bereitstellen von im Behandlungskontext anfallenden Daten von Patient*innen und einen verbesserten Datenaustausch zwischen den Gesundheitsdienstleistern die Behandlung von Patient*innen verbessern und die medizinische Forschung stärken [15].

Zu diesem Zweck werden seit 2018 in einer auf fünf Jahre angelegten Aufbau- und Vernetzungsphase sogenannte Datenintegrationszentren (DIZ) an den deutschen Universitätsklinken etabliert, deren Aufgabe es $u$. a. ist, die in der medizinischen Versorgung und Forschung anfallenden Daten zu strukturieren und auf Standardterminologien abzubilden. Des Weiteren werden die Universitätskliniken über diese neu geschaffene Schnittstelle der DIZ miteinander vernetzt, um die Daten in einer datenschutzkonformen Weise für die standortübergreifende Forschung bereitzustellen. Die vier vom BMBF im Rahmen der MII geförderten Konsortien, DIFUTURE, HiGHmed, MIRACUM und SMITH, entwickeln anhand von vorgegebenen Anwendungsszenarien jeweils unterschiedliche IT-Lösungen, um diesen krankenhausübergreifenden Forschungsdatenaustausch zu ermöglichen [16].

Am Beginn der Förderphase wurde unter Koordination der für Interoperabilität zuständigen MII-Arbeitsgruppe ein Kerndatensatz als Mindestanforderung für die Forschungsdatensätze zu stationären Patient*innen von Vertreter*innen aller vier Konsortien entworfen und abgestimmt. Die Basismodule des Kerndatensatzes beinhalten neben demographischen und administrativen Datenelementen auch Informationen zu Diagnosen, Prozedu- 
ren, Laborbefunden und Medikation. In Erweiterungsmodulen werden zusätzliche Datenelemente definiert, die z.B. für die Einwilligung von Patient*innen oder bildgebende Verfahren eine Rolle spielen oder beispielsweise Bioproben oder onkologische Informationen umfassen [17]. Hinsichtlich der Einwilligung von Patient*innen einigten sich alle Universitätskliniken auf ein einheitliches Einwilligungsverfahren mit einer breiten Beschreibung der Datennutzung („broad consent ${ }^{\prime \prime}$ ), bei welchem Patient*innen selbstbestimmt entscheiden können, in welchem Ausmaß Daten für die medizinische Forschung genutzt werden dürfen [18].

Im 3. MII-Projektjahr wurde im Kontext der Corona-Pandemie das vom BMBF geförderte "Netzwerk Universitätsmedizin" (NUM) gegründet [19]. Vor dem Hintergrund eines verbesserten Datenaustausches wurde der standardisierte GECCODatensatz ("German Corona Consensus") auf Grundlage des MII-Kerndatensatzes definiert, um die technischen und inhaltlichen Voraussetzungen für den Datenaustausch zu an COVID-19 Erkrankten in Deutschland zu schaffen [20].

Die Bereitstellung der im MII-Kerndatensatz abgebildeten Datenelemente durch alle Universitätskliniken bis spätestens zum Ende der aktuellen Förderphase im Dezember 2022 ist ein wichtiger Meilenstein für die Interoperabilität zwischen den Krankenhäusern, der die universitäre Forschung deutlich bereichern wird [21].

\section{Nationale Pandemie Kohorten Netz (NAPKON)}

NAPKON ist eines von 13 Verbundprojekten des NUM und erfasst als nationale prospektive Kohortenstudie umfangreiche,

\section{Autor}

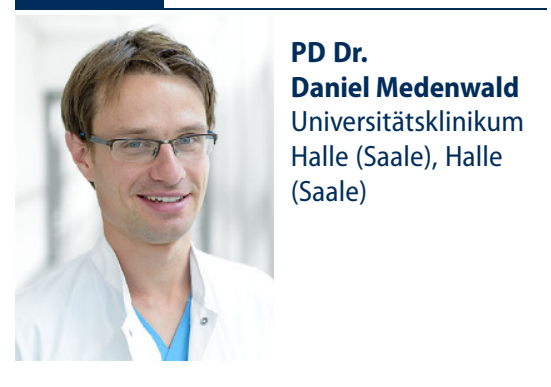

qualitätsgesicherte Daten, Bilddaten und Bioproben von COVID-19-Patient*innen. Damit lassen sich Analysen zum Geschehen der Pandemie in der Onkologie realisieren. Dabei zeichnet NAPKON sich durch die Verbindung einer sektorenübergreifenden Rekrutierung von Patient*innen mit populationsbasierten Ansätzen für die detaillierte Charakterisierung des Akutund Langzeitverlaufes aus. Diese Rekrutierung erfolgt an fast allen deutschen Universitätsklinken sowie an regionalen nichtuniversitären Krankenhäusern und bei niedergelassenen Ärzt*innen. An der Studie beteiligen sich mittlerweile über 60 Standorte und 26 Fach- und Organspezifische Arbeitsgruppen (FOSA), z.B. im Bereich der Onkologie, die FOSA Strahlentherapie sowie Hämatologie und Onkologie, mit insgesamt über 1000 involvierten Personen. Die Onkologie ist dabei in wichtigen Gremien der NAPKON vertreten und damit an zentralen Entscheidungsprozessen beteiligt. Dieses wird koordiniert durch vier Hauptinfrastrukturkerne und drei Kohortenplattformen. Die Kerne dienen insbesondere der Gesamtkoordination des Projektes, der Organisation der Governance, der Qualitätssicherung und Aufbereitung der Rohdaten sowie dem Aufbau einer harmonisierten Biobankplattform. Die in den FOSA organisierten Expert*innengremien agieren im gemeinsamen Fachbeirat auf interdisziplinärer Ebene und gestalten die Studie aktiv mit. Interessierte Wissenschaftler*innen können die gesammelten Daten und Bioproben der aktuell 3204 Patient*innen (Stand 13.09.2021) in einem offenen Use \& Access-Verfahren, auch von außerhalb des Projektes, beantragen. Die umfangreiche Infrastruktur von NAPKON soll in Zukunft über COVID-19 hinaus bestehen bleiben und zur Erforschung weiterer, ggf. neuer Pandemien beitragen.

\section{Fazit für die Praxis}

- Mit der Neustrukturierung der Krebsregister und dem Ausbau weiterer klinischer Datenquellen erschließen sich neue Möglichkeiten für die Versorgungsforschung in Deutschland.

- In Kombination mit prospektiven Studien werden die genannten Datenquellen das Gesicht dieses Forschungsbereiches maßgeblich prägen und gestalten.

\section{Korrespondenzadresse}

\section{PD Dr. Daniel Medenwald}

Klinik und Poliklinik für Strahlentherapie, Universitätsklinikum Halle (Saale)

Ernst-Grube-Straße 40, 06120 Halle (Saale), Deutschland

daniel.medenwald@uk-halle.de

\section{Einhaltung ethischer Richtlinien}

Interessenkonflikt. D. Medenwald, A. SchmidtPokrzywniak, L.A. Kapsner, N. Buttmann-Schweiger, S. Hopff, H. Schmidt und D. Vordermark geben an, dass kein Interessenkonflikt besteht.

Für diesen Beitrag wurden von den Autoren keine Studien an Menschen oder Tieren durchgeführt. Für die aufgeführten Studien gelten die jeweils dort angegebenen ethischen Richtlinien.

\section{Literatur}

1. Kaduszkiewicz $\mathrm{H}$, Teichert $\mathrm{U}$, van den Bussche $H$ (2018) Ärztemangel in der hausärztlichen Versorgung auf dem Lande und im Öffentlichen Gesundheitsdienst. Bundesgesundheitsblatt Gesundheitsforschung Gesundheitsschutz 61(2):187-194

2. Haun MW, Sklenarova H, Zimmermann-Schlegel V, Herzog W, Hartmann M (2018) Psychoonkologische Versorgung im ländlichen Raum. Bundesgesundheitsblatt Gesundheitsforschung Gesundheitsschutz 61(1):89-97

3. Hermes-Moll K, Klein A, Schmitz S, Baumann W (2020) Ergebnisse einer Pilotstudie zur Erfassung von Qualitätsindikatoren in der ambulanten onkologischen Versorgung. Gesundheitswesen 82(01):63-71

4. Pfaff $H$, Glaeske $G$, Neugebauer E, Schrappe M, Zeike S, Schwartz FW et al (2017) Lehrbuch Versorgungsforschung Systematik - Methodik Anwendung, 2. Aufl. (462 Seiten)

5. Deutsches Netzwerk Versorgungsforschung e. V. Memorandum der DNVF-Fachgruppe „Versorgungsforschung in der Onkologie" Kurzfassung. https://www.dnvf.de/files/theme_files/pdf/ PDF-Publikationen/Memorandum\%200nko-VF_ final_kurz.pdf.Zugegriffen:17.Sept. 2021

6. Niederberger M, Peter L (2018) Mixed-MethodsStudien in den Gesundheitswissenschaften. Ein kritischer Überblick. Z Evid Fortbild Qual Gesundhwes 133:9-23

7. Mayring P (2014) Qualitative content analysis: theoretical foundation, basic procedures and software solution. Klagenfurt, S 143. https:// www.ssoar.info/ssoar/handle/document/39517. Zugegriffen: 12.0kt. 2021

8. Craig P, Dieppe P, Macintyre S, Michie S, Nazareth I, Petticrew M (2013) Developing and evaluating complex interventions: the new Medical Research Council guidance. Int J Nurs Stud 50(5):587-592

9. Rahm Hallberg I, Richards DA (2015) Complex interventions in health an overview of research methods. Routledge, London New York (381 Seiten)

10. CFIR Research Team-Center for Clinical Management Research Constructs-the consolidated 
framework for implementation research. https:// cfirguide.org/.Zugegriffen:9.Sept. 2021

11. Bundesanzeiger Gesetz zum Zusammenführen von Krebsregisterdaten. https://www.bgbl.de/ xaver/bgbl/start.xav?startbk=Bundesanzeiger_ BGBI\&jumpTo=bgbl121s3890.pdf\#_bgbl_\%2F $\% 2 F * 5$ B\% 40 attr_id\%3D\%27bgbl121s3890.pdf \%27\%5D_1631388710605.Zugegriffen: 9. Sept. 2021

12. Robert Koch-Institut (2021) Stellungnahme der Ständigen Impfkommission zu Impfungen von Personal in medizinischen Einrichtungen in Deutschland. Epidemiol Bull. https://doi.org/10. 25646/7654

13. Robert Koch-Insitut (2021) Neue Daten, neue Aufgaben: Beim ZfKD werden künftig auch klinische Daten aus den Krebsregistern zusammengeführt. https://www.krebsdaten.de/ Krebs/DE/Content/Publikationen/Kurzbeitraege/ Kurzbeitraege_node.html. Zugegriffen: 10. Sept. 2021

14. Bundesministerium für Gesundheit Bekanntmachung Aktualisierter einheitlicher onkologischer Basisdatensatz der Arbeitsgemeinschaft Deutscher Tumorzentren e.V. (ADT) und der Gesellschaft der epidemiologischen Krebsregister in Deutschland e.V. (GEKID). https://www.gekid.de/ download/1846/.Zugegriffen: 9. Sept. 2021

15. Semler S, Wissing F, Heyder R (2018) German medical Informatics initiative: a national approach to integrating health data from patient care and medical research. Methods Inf Med 57(01):e50-e56

16. Medizininformatik-Initiative Konsortien. https://www.medizininformatik-initiative.de/ de/konsortien. Zugegriffen: 1.Sept. 2021

17. Medizininformatik-Initiative Der Kerndatensatz der Medizininformatik-Initiative. https:// www.medizininformatik-initiative.de/de/derkerndatensatz-der-medizininformatik-initiative. Zugegriffen: 1.Sept. 2021

18. Medizininformatik-Initiative Mustertext zur Patienteneinwilligung. https://www. medizininformatik-initiative.de/de/mustertextzur-patienteneinwilligung. Zugegriffen: 1. Sept. 2021

19. Netzwerk Universitätsmedizin Aufgaben und Ziele. https://www.netzwerk-universitaetsmedizin. de/aufgaben-und-ziele.Zugegriffen: 1.Sept. 2020

20. Sass J, Bartschke A, Lehne M, Essenwanger A, RinaldiE, RudolphSetal (2020)The German Corona Consensus Dataset (GECCO): a standardized dataset for COVID-19 research in university medicine and beyond. BMC Med Inform Decis Mak 20(1):341

21. Medenwald D, Brunner T, Christiansen $H$, Mansoorian S, Vordermark D, Kisser U et al (2021) Shift of radiotherapy use during the first wave of the COVID-19 pandemic? An analysis of German inpatient data. Strahlenther Onkol (under review)

\section{Oncological health services research in Germany. New perspectives through networking}

Apart from prospective health services research with a qualitative and quantitative approach, retrospective data sources are essential for the evaluation of processes in the healthcare system. In Germany, changes in the structure of cancer registries and the establishment of new data sources enable interesting possibilities for health services research. The medical informatics initiative, a network of clinical institutions, has been constructed with which rapid, cross-sectoral and real-life analyses can be accomplished. The restructuring of cancer registries in Germany simplifies population-based studies in the field of oncology and detail of the data could be increased. Based on the newly formed German National Pandemic Cohort Network (NAPKON), the sequelae of the coronavirus disease 2019 (COVID-19) pandemic in the field of oncology can be depicted by a consolidation of university and non-university healthcare providers. By integrating relevant players in the public health system and by considering prospective studies, a comprehensive picture of oncological public health services in Germany can be drawn.

\section{Keywords}

Healthcare system - Cancer registries - Medical informatics initiative - German National Pandemic Cohort Network · COVID-19. Health services research 